\title{
Analyst
}

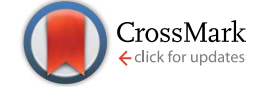

Cite this: Analyst, 2014, 139, 5499

Received 30th July 2014

Accepted 3rd September 2014

DOI: 10.1039/c4an01401d

www.rsc.org/analyst

\section{Sensing with nanopores - the influence of asymmetric blocking on electrochemical redox cycling current $\uparrow$}

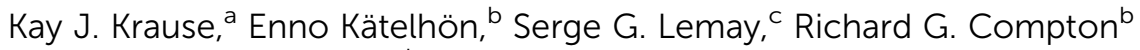 \\ and Bernhard Wolfrum*ad
}

Nanoporous redox cycling devices are highly efficient tools for the electrochemical sensing of redox-active molecules. By using a redox-active mediator, this concept can be exploited for the detection of molecular binding events via blocking of the redox cycling current within the nanopores. Here, we investigate the influence of different blocking scenarios inside a nanopore on the resulting redox cycling current. Our analysis is based on random walk simulations and finite element calculations. We distinguish between symmetric and asymmetric pore blocking and show that the current decrease is more pronounced in the case of asymmetric blocking reflecting the diffusion-driven pathway of the redox-active molecules. Using random walk simulations, we further study the impact of pore blocking in the frequency domain and identify relevant features of the power spectral density, which are of particular interest for sensing applications based on fluctuation analysis.

\section{Introduction}

Lab on a chip devices based on nanoporous structures are promising tools for the detection of molecules. Such devices feature for example nanoscaled pores, which are embedded into a membrane and are exposed to an electrolyte solution. When a molecule enters a pore, an increase of the electrical resistance of the membrane can be measured similar to the Coulter principle. ${ }^{1-3}$ Furthermore, these pores can be modified with certain receptor molecules to increase the selectivity towards specific analytes. ${ }^{4,5}$ Another approach for sensing applications using nanoscaled pores is the detection of an electrochemical current. Here, a part of the pore surface is made of a conducting material and is used as an electrode, which can be biased to a certain potential. Depending on the applied potential, redox-active molecules can react with the electrode generating a Faradaic current. To improve the sensitivity of such a measurement, two individually biased, closely separated electrodes can be implemented in the device. If these electrodes are set to appropriate potentials, redox-active molecules can participate in repetitive

${ }^{a}$ Institute of Bioelectronics (PGI-8/ICS-8) and JARA-Fundamentals of Future Information Technology, Forschungszentrum Jülich, 52425 Jülich, Germany. E-mail: b.wolfrum@fz-juelich.de

${ }^{b}$ Department of Chemistry, Physical and Theoretical Chemistry Laboratory, Oxford University, South Parks Road, Oxford OX1 3QZ, UK

${ }^{c} M E S A+$ Institute for Nanotechnology, University of Twente, PO Box 217, $7500 A E$ Enschede, The Netherlands

${ }^{d}$ Institute of Physics, RWTH Aachen University, 52074 Aachen, Germany

$\dagger$ Electronic supplementary information (ESI) available. See DOI: 10.1039/c4an01401d redox reactions leading to an amplification of the faradaic current. In principle, chip-based redox-cycling sensors can feature different geometries, which all comprise at least two electrodes. For example, different device architectures including interdigitated electrodes, ${ }^{\mathbf{6}-10}$ nanogaps, ${ }^{\mathbf{1 1 - 1 3}}$ nanocavities, $^{\mathbf{1 4 , 1 5}}$ nanochannels ${ }^{16,17}$ or micro- and nanoporous ${ }^{18-30}$ structures have been introduced within the last decades. In particular, nanoporous redox-cycling sensors can be used for the detection of specific binding events within the nanoporous structure. In this case, redox-active molecules may be used as a tracer for electrochemical sensing. Once analyte molecules bind to a specific target within the nanopore, this will lead to a decrease of the redox cycling current due to physical blocking of the pore.

In this paper we investigate the influence of different blocking scenarios on the diffusion-driven redox cycling current and its power spectral density. Specifically, we simulate the redox-cycling current dependence on the blocked area and compare the results for a symmetric and an asymmetric pore blocking. We show that, within a certain range of parameters, asymmetric blocking leads to a stronger decrease of the redox current compared to a symmetric blocking scenario. While finite element simulations can adequately predict the concentration distribution and average currents of the electrochemical sensor, they do not provide insight into the fluctuations of the signal. To address this issue we employ a random walk simulation and investigate the power spectral density of the signal in dependence on the pore blocking. Our results allow the identification of frequency regimes that can be used to detect pore blocking independent of the absolute current magnitude. 


\section{Computational methods}

To investigate the influence of a symmetric and an asymmetric blocking, two different scenarios are simulated. The radius of all simulated nanopores is $25 \mathrm{~nm}$ and the height is $200 \mathrm{~nm}$. Above the pore a reservoir with a radius of $50 \mathrm{~nm}$ and a height of $800 \mathrm{~nm}$ is attached. The simulated nanopore designs are shown in Fig. 1. Symmetric blocking is modeled by a circular narrowing of the pore, which originates from the outer rim (see Fig. 1a). Asymmetric blocking is modeled by a partially circular segment originating from one side of the pore (see Fig. 1b). To simulate the influence of the pore symmetry on the redox current, different magnitudes of the blocked areas are simulated. The blocked area $A$ for a circular blocking can be calculated using the radius of the narrowing $r_{\mathrm{n}}$ and the pore radius $r$.

$$
A_{\text {blocked,symmetric }}=\pi\left(r-r_{\mathrm{n}}\right)^{2}
$$

For the asymmetric blocking the blocked area is given by a circular segment, where $d$ is the height of the segment.

$$
A_{\text {blocked,asymmetric }}=r^{2} \arccos \left(1-\frac{d}{r}\right)-\sqrt{2 r d-d^{2}}(r-d)
$$

The blocking ratio is defined by the ratio of the blocked area and the open pore cross section. The blocked volume within the pore can be calculated by a multiplication of the blocked area with the height of the blocking layer, which is $20 \mathrm{~nm}$.

The calculations following the finite element approach were carried out using COMSOL 4.2. The flux of electrons through the electrodes is defined by the Butler-Volmer equation. The transfer rate is assumed to be $k=6 \times 10^{-2} \mathrm{~m} \mathrm{~s}^{-1}$, the transfer coefficient $\alpha=0.5$, the redox potential $E_{0}=350 \mathrm{mV}$ and the temperature $T=300^{\circ} \mathrm{K}$. The electrode potentials were set to $0 \mathrm{~V}$ (bottom electrode) and $500 \mathrm{mV}$ (top electrode).
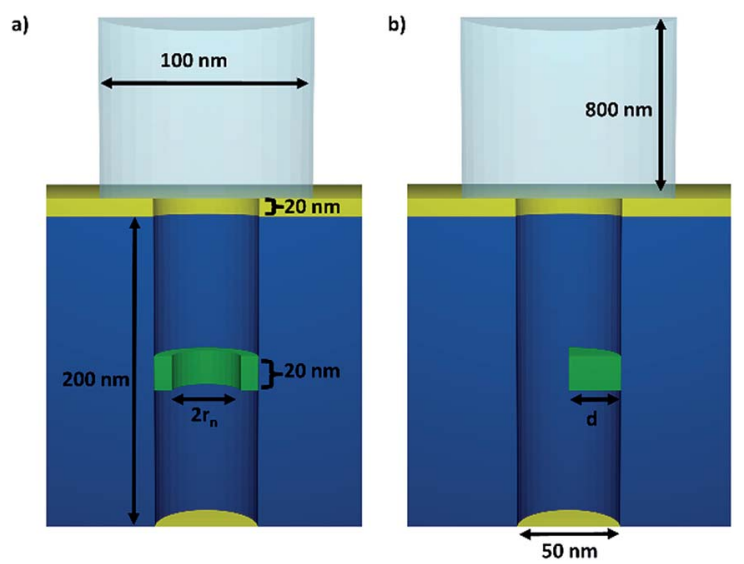

Fig. 1 A sketch of the simulated pore design. The nanopores are 200 $\mathrm{nm}$ high and feature a radius of $25 \mathrm{~nm}$. The blocking region is $20 \mathrm{~nm}$ thick and positioned at a height from 90 to $110 \mathrm{~nm}$. The top electrode is $20 \mathrm{~nm}$ thick and the reservoir is $50 \mathrm{~nm}$ in radius and $800 \mathrm{~nm}$ in height. For a symmetric blocking (a) a cylindrical narrowing with radius $r_{\mathrm{n}}$ is assumed and for an asymmetric (b) blocking a circular segment with segment height $d$.
Finite element methods are useful for the simulation of steady state or transient signals in the time domain. However, they are not suitable for the simulation of current fluctuations, which can provide additional valuable information for sensing applications. The electrochemical correlation spectroscopy for example is based on investigations of the power spectral density of the redox current. ${ }^{31}$ For the calculation of the power spectral density it is necessary to simulate not only the mean value of the current but also the current fluctuations. This can be implemented by random walk simulations, which have previously been used to describe electrochemical sensors. ${ }^{32-36}$ During the random walk, each molecule is randomly displaced for the distance $\mathrm{d} x$ within a certain time step $\mathrm{d} t$. The time step $\mathrm{d} t$ and step width $\mathrm{d} x$ are following the one-dimensional diffusion equation.

$$
\mathrm{d} t=\frac{\mathrm{d} x^{2}}{2 D}
$$

Within each time step the molecules are displaced along all Cartesian axes leading to a total step width of $\sqrt{3} \mathrm{~d} x$. Molecules are reflected upon collisions with boundaries and the redox state is immediately changed by collisions with a properly biased electrode surface. A more detailed description of the simulation software can be found elsewhere. ${ }^{37,38}$ For the simulation of the current decrease due to blocking inside the nanopores, 10 traces are calculated and averaged for each configuration. The calculation of the power spectral density from the simulated current traces is processed in Matlab. Power spectral densities are obtained by averaging over the calculated spectra from 50 traces.

For all calculations the diffusion coefficient of the redox molecules is assumed to be $6.7 \times 10^{-10} \mathrm{~m}^{2} \mathrm{~s}^{-1}$ and the initial concentration of the redox molecules is $c_{\text {red }}=c_{\text {ox }}=\frac{1}{2} c_{0}=150 \mu \mathrm{M}$.

\section{Results and discussion}

We first analyze the current response of the nanoporous sensor for open and partially blocked pores using different configurations. The initial distribution of oxidized and reduced molecules inside the sensor and the reservoir is assumed to be $1: 1$; although the exact distribution has no impact on the steadystate results of the simulation (see $\mathrm{ESI} \dagger$ ).

Exemplary current traces of the bottom electrode derived from the random walk simulation are shown in Fig. 2. As expected, the redox current decreases with an increase of the blocked area. For a blocked area of 15\% (corresponding to 1.5\% volume blocking) this effect is rather small and basically masked by the inherent fluctuations in the signal. For a blocked area of $69 \%$ (blocked volume of $6.9 \%$ ) the current is significantly reduced. Furthermore, an asymmetric blocking condition yields a lower current compared to a symmetric blocking condition. The difference in the current for both blocking scenarios is dependent on the blocked area. To assess this dependence, we simulated the average steady-state redox 


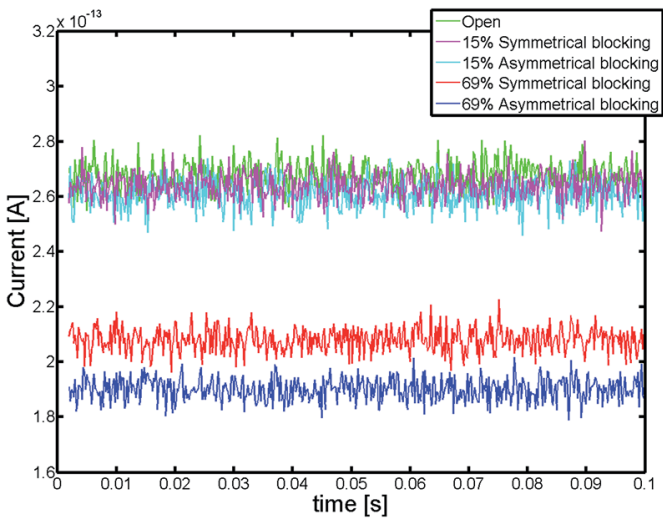

Fig. 2 Redox current traces of the random walk simulation. The currents for an open pore and for a blocking ratio of $15 \%$ and $69 \%$ are shown.

current over a large range of blocking conditions (see Fig. 3) using both, random walk and finite element calculations.

We see that for very small and very large blocking ratios, symmetric and asymmetric currents are similar. However, in between these two extreme scenarios the current decreases more strongly for an asymmetric blocking scenario. In Fig. 3b the difference in the current decrease for the two blocking scenarios is shown. The value is displayed in percentage of the unblocked current at an open pore. At a blocking ratio of about $75 \%$, the difference between symmetrically and asymmetrically blocked currents reaches a maximum. This difference in the currents originates from the diffusive nature of the redox cycling current. The redox active molecules are moving by diffusion and for such processes the time scales with the square of the distance. Therefore, the current is very sensitive to changes in the length of the diffusive pathway, which is in

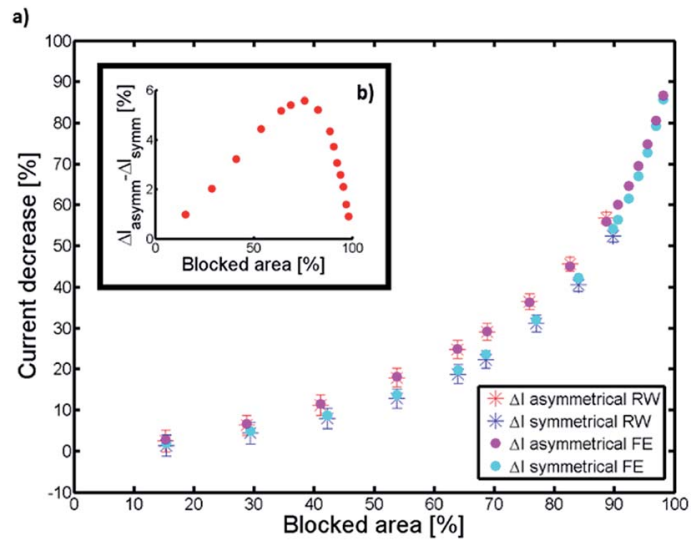

Fig. 3 The mean decrease of the redox current in comparison to an open pore for different blocking ratios and a symmetric and asymmetric pore blocking. The dots represent the data from finite element simulations and the stars the data resulting from the random walk simulations. Each data point is calculated from the mean value of 10 averaged current traces. (b) shows the difference between the current decrease for both blocking scenarios and equal blocked areas using finite element calculations.

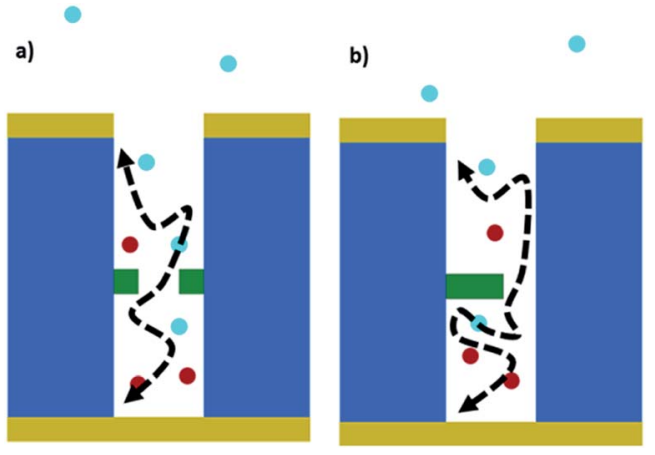

Fig. 4 Schematic cross section of the different blocking methods and their impact on the diffusive pathway of the molecules.

average longer for an asymmetric blocking as for a symmetric blocking (see schematic in Fig. 4).

The results for the mean value of the redox current (Fig. 3) from finite element simulations (dots) are in close agreement with the results from the random walk simulations (stars). However, it seems that for a symmetric blocking the random walk underestimates the current decrease, especially for a high blocking ratio. This is due to the discrete nature of our random walk simulation. The circular pore narrowing is modeled by cubic volume blocks. This approach is only valid if $\mathrm{d} x \gg r_{\mathrm{n}}$. Therefore, the results from the random walk differs from the results of the finite element calculations for very high blocking ratios.

We further analyzed the effect of blocking on the signal fluctuation. In Fig. 5, the power spectral density (PSD) of the redox current is shown for an open pore and an area blocking ratio of $69 \%$ and $89 \%$ respectively $90 \%$.

The power spectral density features a plateau at low frequencies and decays with a power law after a transition frequency of approximately $1 \mathrm{kHz}$. For higher frequencies around $6 \mathrm{kHz}$ a second plateau appears. The shape of the power spectral density for low frequencies is dominated by the fluctuating number of particles inside the pore. This behavior is

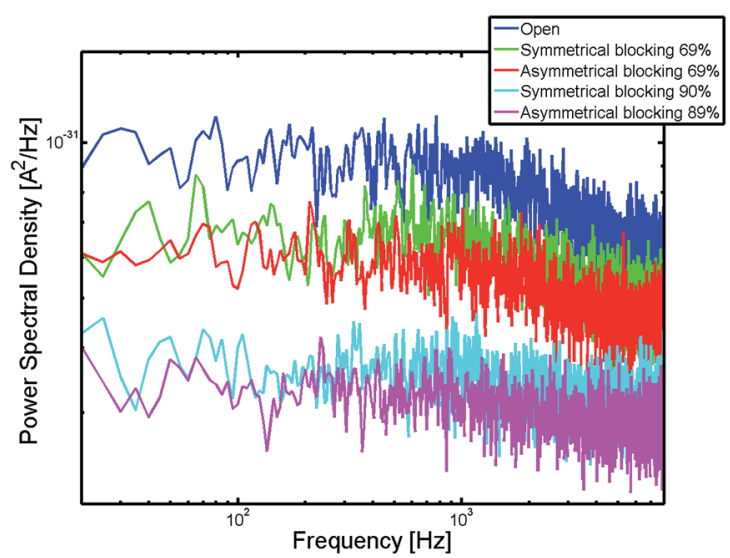

Fig. 5 The power spectral density of the redox current generated with random walk simulations. 


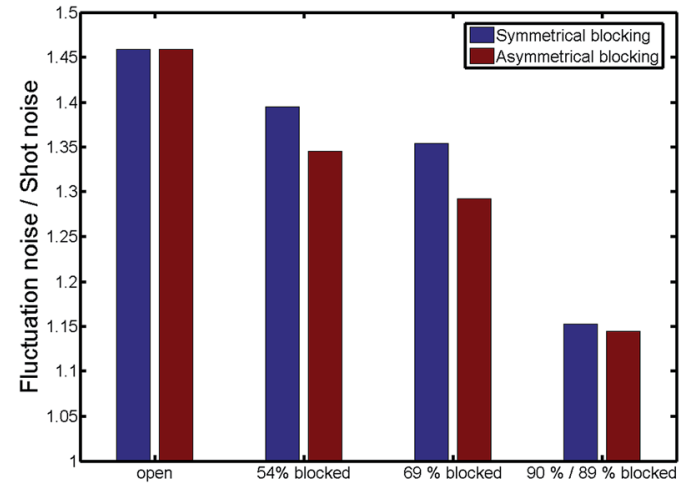

Fig. 6 The ratio of the absolute values of the fluctuation and shot noise plateaus. The spectra are averaged from $10 \mathrm{~Hz}$ to $1 \mathrm{kHz}$ for the fluctuation noise and from $6 \mathrm{kHz}$ to $8 \mathrm{kHz}$ for the shot noise.

known from other nanoelectrochemical devices such as nanofluidic channels $^{31}$ or nanocavities. ${ }^{37}$ The fluctuation noise features a plateau at low frequencies and decays with a power law after a transition frequency $f_{0}$. The second plateau at high frequencies is a shot-like noise and caused by the diffusive movement of the molecules in between the electrodes. ${ }^{37}$ With an increase of the blocking ratio, we see that the spectra are shifted to lower values. Additionally, the difference between the first and second plateau decreases. The ratio of the mean values of these plateaus is shown in Fig. 6 .

Both, fluctuation noise and shot-like noise scale linearly with the number of redox molecules. Consequently, the ratio of the plateaus is a current independent parameter, which could be used for future sensing applications, assuming that the currents are not masked by thermal noise or other interfering signals. The effect of blocking on this parameter due to a particle of $3 \mathrm{~nm}$ radius for two pore geometries $(15 \mathrm{~nm}$ and 50 nm diameter) is shown in the ESI. $\dagger$

\section{Conclusions}

We calculated the decrease of the faradaic redox cycling current within a nanopore caused by symmetric and asymmetric blocking of the pore. Using finite element calculations and random walk simulations we demonstrated that the current is suppressed more strongly for an asymmetric blocking scenario. This effect is caused by the diffusion time of the molecules between the electrodes, which is sensitive to the square of the diffusive pathway. Furthermore, we analyzed the influence of the pore blocking on the power spectral density of the signal. The difference of two plateaus in the spectrum, caused by fluctuations and shot-like noise, decreases with an increasing blocking ratio. The ratio of the two signals in the frequency domain is independent of the absolute current and can potentially be used as a parameter for future sensing applications.

\section{Acknowledgements}

We gratefully acknowledge funding by the Helmholtz Young Investigators Program.

\section{Notes and references}

1 C. Dekker, Nat. Nanotechnol., 2007, 2, 209-215.

2 D. Branton, D. W. Deamer, A. Marziali, H. Bayley, S. A. Benner, T. Butler, M. Di Ventra, S. Garaj, A. Hibbs, X. Huang, S. B. Jovanovich, P. S. Krstic, S. Lindsay, X. S. Ling, C. H. Mastrangelo, A. Meller, J. S. Oliver, Y. V. Pershin, J. M. Ramsey, R. Riehn, G. V. Soni, V. Tabard-Cossa, M. Wanunu, M. Wiggin and J. A. Schloss, Nat. Biotechnol., 2008, 26, 1146-1153.

3 S. Howorka and Z. Siwy, Chem. Soc. Rev., 2009, 38, 2360.

4 S.-J. Li, J. Li, K. Wang, C. Wang, J.-J. Xu, H.-Y. Chen, X.-H. Xia and Q. Huo, ACS Nano, 2010, 4, 6417-6424.

5 R. Wei, V. Gatterdam, R. Wieneke, R. Tampé and U. Rant, Nat. Nanotechnol., 2012, 7, 257-263.

6 D. G. Sanderson and L. B. Anderson, Anal. Chem., 1985, 57, 2388-2393.

7 P. Van Gerwen, W. Laureyn, W. Laureys, G. Huyberechts, M. Op De Beeck, K. Baert, J. Suls, W. Sansen, P. Jacobs, L. Hermans and R. Mertens, Sens. Actuators, B, 1998, 49, 73-80.

8 V. A. T. Dam, W. Olthuis and A. van den Berg, Analyst, 2007, $132,365$.

9 L. Sasso, A. Heiskanen, F. Diazzi, M. Dimaki, J. Castillo-León, M. Vergani, E. Landini, R. Raiteri, G. Ferrari, M. Carminati, M. Sampietro, W. E. Svendsen and J. Emnéus, Analyst, 2013, 138, 3651-3659.

10 K. Ino, T. Nishijo, Y. Kanno, F. Ozawa, T. Arai, Y. Takahashi, H. Shiku and T. Matsue, Electrochemistry, 2013, 81, 682-687.

11 S. Strobel, K. Arinaga, A. Hansen and M. Tornow, Nanotechnology, 2007, 18, 295201.

12 S. Harrer, S. Strobel, G. Penso Blanco, G. Scarpa, G. Abstreiter, M. Tornow and P. Lugli, IEEE Trans. Nanotechnol., 2009, 8, 662-670.

13 M. J. J. van Megen, J. G. Bomer, W. Olthuis and A. van den Berg, Microelectron. Eng., 2014, 115, 21-25.

14 E. Kätelhön, B. Hofmann, S. G. Lemay, M. A. G. Zevenbergen, A. Offenhäusser and B. Wolfrum, Anal. Chem., 2010, 82, 8502-8509.

15 M. G. Straver, M. Odijk, W. Olthuis and A. van den Berg, Lab Chip, 2012, 12, 1548-1553.

16 B. Wolfrum, M. Zevenbergen and S. Lemay, Anal. Chem., 2008, 80, 972-977.

17 M. A. G. Zevenbergen, P. S. Singh, E. D. Goluch, B. L. Wolfrum and S. G. Lemay, Nano Lett., 2011, 11, 28812886.

18 Z. P. Aguilar, W. R. Vandaveer and I. Fritsch, Anal. Chem., 2002, 74, 3321-3329.

19 W. R. Vandaveer IV, D. J. Woodward and I. Fritsch, Electrochim. Acta, 2003, 48, 3341-3348.

20 S. Neugebauer, U. Müller, T. Lohmüller, J. P. Spatz, M. Stelzle and W. Schuhmann, Electroanalysis, 2006, 18, 1929-1936.

21 T. Lohmüller, U. Müller, S. Breisch, W. Nisch, R. Rudorf, W. Schuhmann, S. Neugebauer, M. Kaczor, S. Linke, S. Lechner, J. Spatz and M. Stelzle, J. Micromech. Microeng., 2008, 18, 115011. 
22 S. Neugebauer, L. Stoica, D. Guschin and W. Schuhmann, Microchim. Acta, 2008, 163, 33-40.

23 D. Menshykau, A. M. O'Mahony, F. J. del Campo, F. X. Munõz and R. G. Compton, Anal. Chem., 2009, 81, 9372-9382.

24 D. Menshykau, M. Cortina-Puig, F. J. del Campo, F. X. Muñoz and R. G. Compton, J. Electroanal. Chem., 2010, 648, 28-35.

25 F. Zhu, J. Yan, M. Lu, Y. Zhou, Y. Yang and B. Mao, Electrochim. Acta, 2011, 56, 8101-8107.

26 S. P. Branagan, N. M. Contento and P. W. Bohn, J. Am. Chem. Soc., 2012, 134, 8617-8624.

27 C. Ma, N. M. Contento, L. R. Gibson and P. W. Bohn, Anal. Chem., 2013, 85, 9882-9888.

28 M. Hüske, R. Stockmann, A. Offenhäusser and B. Wolfrum, Nanoscale, 2013, 6, 589-598.

29 Y. Lim, J.-I. Heo and H. Shin, Sens. Actuators, B, 2014, 192, 796-803.

30 M. Hüske, A. Offenhäusser and B. Wolfrum, Phys. Chem. Chem. Phys., 2014, 16, 11609-11616.
31 M. A. G. Zevenbergen, P. S. Singh, E. D. Goluch, B. L. Wolfrum and S. G. Lemay, Anal. Chem., 2009, 81, 8203-8212.

32 S. Licht, V. Cammarata and M. S. Wrighton, Science, 1989, 243, 1176-1178.

33 G. Nagy, Y. Sugimoto and G. Denuault, J. Electroanal. Chem., 1997, 433, 167-173.

34 R. J. White and H. S. White, Langmuir, 2008, 24, 2850-2855.

35 I. J. Cutress, E. J. F. Dickinson and R. G. Compton, J. Electroanal. Chem., 2011, 655, 1-8.

36 E. Kätelhön, K. J. Krause, B. Wolfrum and R. G. Compton, ChemPhysChem, 2014, 15, 872-875.

37 E. Kätelhön, K. J. Krause, P. S. Singh, S. G. Lemay and B. Wolfrum, J. Am. Chem. Soc., 2013, 135, 8874-8881.

38 E. Kätelhön, K. J. Krause, K. Mathwig, S. G. Lemay and B. Wolfrum, ACS Nano, 2014, 8, 4924-4930. 